\title{
A persistent ultraviolet outflow from the accretion disc in a transient neutron star binary
}

Noel Castro Segura ( $D$ N.Castro-Segura@soton.ac.uk)

University of Southampton https://orcid.org/0000-0002-5870-0443

Christian Knigge

University of Southampton

Knox Long

Space Telescope Science Institute https://orcid.org/0000-0002-4134-864X

Diego Altamirano

Southampton University https://orcid.org/0000-0002-3422-0074

Montserrat Armas Padilla

IAC

Charles Bailyn

Yale University

David Buckley

South African Astronomical Observatory https://orcid.org/0000-0002-7004-9956

Douglas Buisson

University of Southampton

Jorge Casares

Instituto de Astrofísica de Canarias https://orcid.org/0000-0001-5031-0128

Phil Charles

University of Southampton

Jorge Combi

CONICET

Virginia A. Cúneo

IAC

Nathalie Degenaar

University of Amsterdam https://orcid.org/0000-0002-0092-3548

Santiago del Palacio

CONICET

Maria Diaz Trigo

ESO

Rob Fender

University of Oxford

Poshak Gandhi 
University of Southampton

\section{Claudia Gutíerrez}

University of Southampton

\section{Juan Hernández Santisteban}

University of St Andrews https://orcid.org/0000-0002-6733-5556

\section{Felipe Jiménez lbarra}

IAC

\section{James Matthews}

University of Cambridge

\section{Mariano Mendez}

University of Groningen https://orcid.org/0000-0003-2187-2708

\section{Matthew Middleton}

University of Southampton

\section{Teo Muñoz Darias}

IAC

\section{Mehtap Özbey Arabaci}

University of Southampton

\section{Mayukh Pahari}

University of Southampton

\section{Lauren Rhodes}

University of Oxford

\section{Thomas Russell}

Anton Pannekoek Instituut voor Sterrenkunde https://orcid.org/0000-0002-7930-2276

\section{Simone Scaringi}

University of Durham

Jakob van den Eijden

University of Oxford

Georgios Vasilopolulos

University of Yale

Federico Vincentelli

University of Southampton

Phil Wiseman

University of Southampton

\section{Physical Sciences - Article}

Keywords: accretion disc, Swift J1858.6-0814, blue-shifted absorption

Posted Date: July 21st, 2021 
DOI: https://doi.org/10.21203/rs.3.rs-701353/v1

License: (c) (1) This work is licensed under a Creative Commons Attribution 4.0 International License. Read Full License

Version of Record: A version of this preprint was published at Nature on March 2nd, 2022. See the published version at https://doi.org/10.1038/s41586-021-04324-2. 


\title{
A persistent ultraviolet outflow from the accretion disc in a transient neutron star binary
}

\author{
N. Castro Segura ${ }^{1, \star}$, C. Knigge ${ }^{1}$, K.S. Long ${ }^{2,3}$, D. Altamirano ${ }^{1}$, M. Armas Padilla ${ }^{4,5}$, \\ C. Baylin ${ }^{6}$, D.A.H. Buckley ${ }^{7}$, D.J.K. Buisson ${ }^{1}$, J. Casares ${ }^{4,5}$, P. Charles $^{1}$, J.A. Combi ${ }^{8}$, \\ V.A. Cúneo ${ }^{4,5}$, N.D. Degenaar ${ }^{9}$, S. del Palacio ${ }^{8}$, M. Díaz Trigo $^{10}$, R. Fender ${ }^{11}$, P. Gandhi ${ }^{1}$, \\ C. Gutíerrez ${ }^{1,12,13}$, J. V. Hernandez Santisteban ${ }^{14}$, F. Jiménez Ibarra ${ }^{4,5,15,16}$, J. Matthews ${ }^{17}$, \\ M. Méndez ${ }^{18}$, M. Middleton ${ }^{1}$, T. Muñoz-Darias ${ }^{4,5}$, M. Özbey Arabacı ${ }^{1,19}$, M. Pahari ${ }^{1,20}$, \\ L. Rhodes ${ }^{11}$, T.D. Russell ${ }^{9,21}$, S. Scaringi ${ }^{22}$, J. van den Eijnden ${ }^{9,11}$, G. Vasilopoulos ${ }^{6,23}$, \\ F.M. Vincentelli ${ }^{1}$, and P. Wiseman ${ }^{1}$
}

\footnotetext{
${ }^{1}$ Department of Physics \& Astronomy. University of Southampton, Southampton SO17 1BJ, UK

${ }^{2}$ Space Telescope Science Institute, 3700 San Martin Drive, Baltimore, MD 21218, USA

${ }^{3}$ Eureka Scientific, Inc. 2452 Delmer Street, Suite 100, Oakland, CA 94602-3017, USA

${ }^{4}$ Instituto de Astrofísica de Canarias, E-38205 La Laguna, Tenerife, Spain

${ }^{5}$ Departamento de Astrofísica, Universidad de La Laguna, E-38206 La Laguna, Tenerife, Spain

${ }^{6}$ Department of Astronomy, Yale University, PO Box 208101, New Haven, CT 06520-8101, USA

${ }^{7}$ South African Astronomical Observatory, PO Box 9, Observatory 7935, Cape Town, South Africa

${ }^{8}$ Instituto Argentino de Radioastronomía (CONICET;CICPBA;UNLP), C.C. No 5, 1894, Villa Elisa, Argentina

${ }^{9}$ Anton Pannekoek Institute for Astronomy, University of Amsterdam, Science Park 904, 1098 XH Amsterdam, The

Netherlands

${ }^{10}$ ESO, Karl-Schwarzschild-Strasse 2, D-85748 Garching bei, München, Germany

${ }^{11}$ Astrophysics, Department of Physics, University of Oxford, Denys Wilkinson Building, Keble Road, Oxford OX1

$3 \mathrm{RH}, \mathrm{UK}$

${ }^{12}$ Finnish Centre for Astronomy with ESO (FINCA), FI-20014 University of Turku, Finland

${ }^{13}$ Tuorla Observatory, Department of Physics and Astronomy, FI-20014 University of Turku, Finland

${ }^{14}$ SUPA School of Physics \& Astronomy, University of St Andrews, North Haugh, St Andrews KY16 9SS, UK

${ }^{15}$ Department of Physics and Astronomy, Macquarie University, Sydney, NSW 2109, Australia

${ }^{16}$ Research Centre in Astronomy, Astrophysics and Astrophotonics, Macquarie University, Sydney, NSW 2109,

Australia

${ }^{17}$ Institute of Astronomy, University of Cambridge, Madingley Road, Cambridge CB3 OHA, UK

${ }^{18}$ Kapteyn Astronomical Institute, University of Groningen, P.O. BOX 800, 9700 AV Groningen, The Netherlands

${ }^{19}$ Faculty of Science, Department of Astronomy and Space Sciences, Atatürk University, Yakutiye, Erzurum 25240,

Turkey

${ }^{20}$ Department of Physics, IIT Hyderabad, Hyderabad 502285, India

${ }^{21}$ INAF, Istituto di Astrofisica Spaziale e Fisica Cosmica, Via U. La Malfa 153, I-90146 Palermo, Italy

${ }^{22}$ Centre for Extragalactic Astronomy, Department of Physics, Durham University, DH1 3LE, UK

${ }^{23}$ Université de Strasbourg, CNRS, Observatoire astronomique de Strasbourg, UMR 7550, 67000, Strasbourg,

France

*e-mail address: N.Castro-Segura@soton.ac.uk
} 
All disc-accreting astrophysical objects also produce powerful disc winds and/or jets. In compact binaries containing neutron stars or black holes, accretion often takes place during violent outbursts. The main disc wind signatures seen during these eruptions are blue-shifted X-ray absorption lines. However, these signatures are only observed during "soft states", when the accretion disc generates most of the luminosity ${ }^{1}$. By contrast, optical wind-formed absorption lines have recently been detected in "hard states", when the luminosity is dominated by a hot corona ${ }^{2}$. The relationship between these disc wind signatures is unknown, and no erupting compact binary has so far been observed to display wind-formed lines between the X-ray and optical bands, despite the many strong resonance transitions in this ultraviolet (UV) region of the spectrum ${ }^{3}$. In turn, the impact of disc winds on the overall mass and energy budget of these systems remains a key open question. Here, we show that the transient neutron star X-ray binary $\mathbf{S w i f t} \mathbf{J 1 8 5 8 . 6}-\mathbf{0 8 1 4}$ exhibits wind-formed, blue-shifted absorption features associated with $\mathrm{C} \mathrm{IV,} \mathrm{N} \mathrm{V} \mathrm{and} \mathrm{He} \mathrm{II} \mathrm{in} \mathrm{time-resolved,} \mathrm{UV} \mathrm{spectroscopy}$ obtained with the Cosmic Origins Spectrograph on board the Hubble Space Telescope during a luminous hard state. In simultaneous ground-based observations, the optical $\mathrm{H}$ and $\mathrm{He}$ I lines also display transient blue-shifted absorption troughs. By decomposing our UV data into constant and flaring components, we demonstrate that the blue-shifted absorption is associated with the former, which implies that the outflow is always present. The joint presence of UV and optical wind features in the hard state reveals a multi-phase and/or spatially stratified evaporative outflow from the outer disc. This type of persistent mass loss across all accretion states has been predicted by radiation-hydrodynamic simulations ${ }^{4}$ and is required to account for the shorter-than-expected outburst durations ${ }^{5,6}$.

On October 2018, the Neil Gehrels Swift Observatory (Swift ${ }^{7}$ ) detected a bright new X-ray binary transient, Swift J1858.6-0814 (hereafter J1858) ${ }^{8}$. Multi-wavelength observations quickly led to the discovery of radio, optical and nearultraviolet (UV) counterparts ${ }^{9-11}$. The detection of thermonuclear runaway explosions in X-rays (Type I X-ray bursts) established that the accreting object is a neutron star located at a distance of about $13 \mathrm{kpc}^{12}$. The system was also found to undergo eclipses, implying a nearly edge-on viewing angle with respect to the disc $\left(i \gtrsim 70^{\circ}\right)$ and revealing the orbital period to be $P_{\text {orb }} \simeq 21.3 \mathrm{~h}^{13}$.

J1858 displayed extreme variability during its outburst in all energy bands, with the X-ray luminosity changing by 1-2 orders of magnitude on time-scales of seconds (see Figure 1-a ${ }^{13-16}$. The X-ray spectrum consisted of a heavily absorbed thermal accretion disc component plus a very steep non-thermal power law tail (photon flux $N_{p h}(E) \propto E^{-\Gamma}$, with $\left.\Gamma<1\right)^{17,18}$. Both the peculiar X-ray spectrum and spectacular variability are reminiscent of those seen during the outbursts of the well-studied black-hole X-ray binaries V404 Cyg and V4641 Sgr, which are thought to be a consequence of accretion at super-Eddington rates $^{19-22}$.

In order to shed light on the accretion and outflow processes associated with the outburst, we carried out strictly simultaneous, time-resolved observations across the electromagnetic spectrum on August 6, 2019 around 00 (UTC). One of our primary goals was to search for outflow signatures in the far-ultraviolet (far-UV) band, since this region contains several strong resonance lines that are very sensitive to the presence of warm, moderately-ionized intervening material.Therefore, the timing of this campaign was centered on far-UV spectroscopic observations with the Hubble Space Telescope (HST). Simultaneous optical spectroscopy was obtained at both the Very Large Telescope (VLT) array and the Gran Telescopio de Canarias (GTC). Additional information about the campaign is provided in the Extended Data section.

In line with data obtained at other wavelengths ${ }^{10,13,23}$, the far-UV light curve exhibits dramatic flaring activity (Figure 1-b). The X-ray, far-UV and optical variability are clearly correlated, with any lags between these time series being $\lesssim 1$ s (Vincentelli et al. in prep). This suggests that the multi-wavelength flaring is driven by a variable central X-ray source.

The presence of a large, strongly irradiated accretion disc is the key requirement for a thermally-driven outflow ${ }^{24-26}$, while high inclinations tend to strengthen wind-formed absorption features ${ }^{1,4,27}$. All of this makes J1858 an ideal candidate for displaying clear observational outflow signatures. In addition, X-ray spectroscopy of the source obtained earlier in the same outburst revealed redshifted N VII emission, tentatively suggesting that the blue wing of this line was absorbed in an outflow, even though the system was still in the hard state during these observations ${ }^{28}$. Time-resolved optical spectroscopy also revealed clear, but highly variable P Cygni wind features in $\mathrm{H} \alpha$ and He I $5876 \AA$ during the bright hard state ${ }^{2}$ (Figure 2).

Figure 3 shows the time-averaged far-UV spectrum we obtained with HST in the hard state. The spectrum is rich in both absorption and emission lines that span a wide range of ionization states. Most of the low-ionization absorption lines are centered at or near the rest wavelength of the relevant transition, with most of these lines not being intrinsic to the system but rather due to interstellar absorption along the line of sight. However, at least two emission lines $-\mathrm{N}$ V $1240 \AA$ and C IV $1550 \AA$ - show clear evidence for associated blue-shifted absorption. Since these species are associated with temperatures of $T \simeq$ a few $\times 10^{4} \mathrm{~K}$, their presence unambiguously establishes the existence of a warm and moderately ionized outflowing component.

Several other transitions - e.g. O v $1370 \AA$ and Si III $1440 \AA$ - also show tentative evidence for blue-shifted absorption. Moreover, all strong emission lines in the spectrum - which includes the Si IV $1400 \AA$ doublet resonance line and the He II $1640 \AA$ 
recombination feature - show evidence for a slight red-shift or a red-skew, suggesting that they are also affected by blue-shifted wind absorption.

As shown in the insets of Figure 3, the blue edges of the far-UV absorption features extend up to $\simeq-2000 \mathrm{~km} \mathrm{~s}^{-1}$, similar to the wind speed inferred from the optical data. However, the far-UV absorption troughs are considerably deeper than those in the optical, which rarely fall below $90 \%-95 \%$ of the continuum. This is likely because most of the strong far-UV lines are associated with strongly scattering resonance transitions, whereas the optical features are associated with recombination lines that connect two excited levels. Very high (column) densities are required in order for such recombination lines to produce absorption. On the other hand, sensitivity of far-UV resonance lines to intervening material makes this waveband particularly valuable for studying outflows ${ }^{29}$.

In order to establish if the far-UV wind signatures are always present - or are instead associated with the strong flaring events in the light curve - we have carried out a maximum likelihood linear decomposition of the time-resolved spectroscopy into a constant and a flaring (variable) component. The spectra inferred for the two components are shown in Figure 4; details regarding the decomposition technique are provided in the Methods section. In both N V $1240 \AA$ and C IV $1550 \AA$, the blue-shifted absorption signature is clearly associated with the constant component. This suggests that our line of sight to the emitting region resposible for the flaring component does not pass through the warm outflow. Perhaps more importantly, it also suggests that the outflow is, in fact, always present, but that its signatures may sometimes be swamped by the flaring component (in which these signatures are absent). The same effect may be responsible for the transience of the blue-shifted absorption seen in the optical data, especially considering how weak these features are (c.f. Figure 2).

The presence of detectable blue-shifted absorption associated with the UV resonance lines (e.g. N v $1240 \AA$, C IV $1550 \AA$ ) implies that the optical depth in these transitions must be significant. This, in turn, requires minimum column densities for the relevant ions, which can be cast as approximate lower limits on the mass-loss rate carried away by the outflow (see Methods for details). Conservatively assuming ionization fractions of $f=1$ for both $C^{3+}$ and $\mathrm{N}^{4+}$, these limits are $\dot{M}_{\text {wind }} \gtrsim 2 \times 10^{-11} \mathrm{M}_{\odot} y r^{-1}$ for $\mathrm{N} \mathrm{v} 1240 \AA$ and $\dot{M}_{\text {wind }} \gtrsim 3 \times 10^{-12} \mathrm{M}_{\odot} y r^{-1}$ for C IV $1550 \AA$. The actual ionization fractions may be considerably lower, and the mass-loss rate correspondingly higher.

The apparent time-averaged X-ray luminosity during the flaring hard state in which we observed J1858 was $L_{X} \simeq 0.01 L_{\text {Edd }}$, although individual flares appear to have reached super-Eddington levels ${ }^{12}$. Taken at face value, this corresponds to an average accretion rate in this state of $\dot{M}_{\text {acc }} \simeq 10^{-10} \mathrm{M}_{\odot} \mathrm{yr}^{-1}$. In this case, $\dot{M}_{\text {wind }} / \dot{M}_{\text {acc }} \gtrsim 0.2$, suggesting that the wind is dynamically important and could significantly affect the accretion flow ${ }^{30,31}$. However, it is also possible that the intrinsic luminosity was much higher throughout this state, with time-variable obscuration being responsible for the reduction in the time-averaged flux (and perhaps also the flaring activity). In the extreme case that $L \simeq L_{E d d}$, the constraint on the wind efficiency is $\dot{M}_{\text {wind }} / \dot{M}_{\text {acc }} \gtrsim 10^{-3}$.

The discovery of optical, UV and (probably) X-ray outflow signatures in the luminous hard state of J1858 suggests that disc winds may always be present in transient X-ray binaries, not just in disc-dominated soft states. Our identification of the constant (non-flaring) spectral component as the carrier of these signatures in the far-UV strongly supports this idea. X-ray and far-UV wind signatures have also been observed in some persistent soft-state X-ray binaries, ${ }^{32-35}$, i.e. systems in which the disc is not subject to the instability that drives the outbursts of transient accretors ${ }^{36-38}$

The emerging physical picture of disc winds being an integral part of the accretion flows in X-ray binaries is consistent with theoretical modeling of outburst light curves ${ }^{5,37}$. It is also in line with recent radiation-hydrodynamical modeling of thermally-driven outflows from X-ray binary discs ${ }^{4}$. These simulations confirm that strong mass loss is inevitable in any systems with a sufficiently large disc subject to strong irradiation. Both conditions are met in J1858 (see Methods). A key test of the thermally-driven wind scenario will be to check that wind signatures are absent in systems where these conditions are not met $^{39}$.

Regardless of the driving mechanism, two key outstanding questions are where and how these outflows manage to sustain a sufficiently low ionization state to allow the formation of optical and UV lines. The most likely answers are that selfshielding, probably coupled with clumping, protects parts of the dense base of the wind above the outermost disc regions from over-ionization. Radiative transfer modeling will be needed to confirm this ${ }^{3,4,40}$.

\section{Acknowledgements}

NCS \& CK acknowledge support by the Science and Technology Facilities Council (STFC), and from STFC grant ST/M001326/1. Partial support for KSL's effort on the project was provided by NASA through grant numbers HST-GO-15984 and HST-GO16066 from the Space Telescope Science Institute, which is operated by AURA, Inc., under NASA contract NAS 5-26555. ND acknowledges support from a Vidi grant for the Netherlands Organization for Scientific Research (NWO). VAC acknowledges support from the Spanish Ministerio de Ciencia e Innovación under grant AYA2017-83216-P. JM acknowledges a Herchel Smith Fellowship at Cambridge. TDR acknowledge financial contribution from the agreement ASI-INAF n.2017-14-H.0. JvdE is supported by a Lee Hysan Junior Research Fellowship from St Hilda's College, Oxford. GV acknowledges support by NASA 
Grants 80NSSC20K1107, 80NSSC20K0803 and 80NSSC21K0213. MÖA acknowledges support from the Royal Society through the Newton International Fellowship programme.

\section{Author information}

Reprints and permissions information is available at www.nature.com/reprints. The authors declare no competing financial interests. All correspondence should be addressed to N.C.S. (N.Castro-Segura@ Soton.ac.uk)

\section{Methods}

\section{Ultraviolet Spectroscopy Observations}

Swift J1858.6-0814 was observed under the program GO/DD 15984 with N. Castro Segura as PI on Aug 5, 2019 23:49:20 UT. A total of 4.9 kilo seconds (ks) exposure was obtained in the far-UV with the Cosmic Origin Spectrograph $\left(\operatorname{COS}^{41}\right)$ and the G140L grating using the primary science aperture (PSA). This configuration provides a spectral resolution of $\mathrm{R}=\lambda / \Delta \lambda \sim 900$. All the observations were obtained in TIME-TAG mode, yielding a stream of detected events at a time resolution of $32 \mathrm{~ms}$.

\section{Data analysis}

We reduced the far-UV data using the HST CALCOS pipeline ${ }^{1}$. One-dimensional spectra were extracted using the TwOZONE algorithm, which sums over the cross-dispersion direction such that $99 \%$ of the flux is extracted at each wavelength. Errors are estimated from Poisson statistics, and the background is modeled with a smooth polynomial and subtracted from the target spectrum. We extracted light curves from the TIME-TAG event files using the same regions defined by the pipeline, except that empirical background correction was directly applied. Regions affected by geocoronal airglow emission associated with Lyman $\alpha(\lambda \lambda 1208-1225 \AA)$ and O II $(\lambda \lambda 1298-1312 \AA)$ were masked when extracting the light curves.

\section{Spectral decomposition}

The highly variable far-UV luminosity during our observations gives rise to a strongly bimodal logarithmic flux distribution (Extended Data Figure 1). This is in line with the visual impression from the far-UV light curve that the dominant variability is due to "shots" or "flares" superposed on a roughly constant background (Figure 1-b).

In order to isolate the spectra associated with these two components, we have carried out a simple linear decomposition of our time-resolved spectroscopic data into a constant and a flaring (variable) component. Following Eracleous \& Horne $(1996)^{42}$, we assume that the flux density $F(\lambda, t)$ at wavelength $\lambda$ and time $t$ can be written as

$$
F(\lambda, t)=C(\lambda)+V(\lambda) D(t),
$$

where $C(\lambda)$ and $V(\lambda)$ are the spectra of the constant and flaring components, respectively. The function $D(t)$ is the driving light curve of the flaring component.

In order to estimate $D(t)$, we constructed a far-UV continuum light curve at $10 \mathrm{~s}$ time resolution. We then estimated the underlying constant level in this light curve and created a normalized driving light curve from which this estimate was removed. We finally smooth the resulting time series with a 5-point, second-order Savitzky-Golay filter to obtain our estimate of $D(t)$. The result is shown as the red curve in Extended Data Figure 2.

With $D(t)$ fixed, the decomposition described by Equation 1 becomes just a series of $N_{\lambda}$ 2-parameter fits, where $N_{\lambda}$ is the number of wavelength bins being considered. Since HST/COS uses a photon-counting far-UV detector, the data set actually consists of a time- and wavelength-tagged event stream. In order to maximize the signal-to-noise ratio of the inferred spectral components, our decomposition is based on an unbinned (in time) maximum likelihood fit to the data at the individual photon-event level. Detector regions dominated by background and/or geo-coronal emission are excluded from the fit. The spectra described as constant and flaring components in Figure 4 are the resulting maximum-likelihood estimates of $C(\lambda)$ and $V(\lambda)$.

\section{Optical spectroscopy}

During the $H S T$ visit, strictly simultaneous observations of $S$ wift J1858.6-0814 were carried out with $X$-Shooter ${ }^{43}$ (program ID 2103.D-5052(A)) and OSIRIS $^{44}$ spectrographs (program ID GTC23-19A), mounted on the VLT UT2-Kueyen telescope in Paranal Observatory and in GTC at Roque de los Muchachos Observatory, respectively. X-Shoooter yielded time-resolved optical/NIR spectra covering the range $\lambda \lambda 0.3-2.4 \mu \mathrm{m}$. With this instrument we obtained a total of 58 individual exposures with integration times of $\simeq 300 \mathrm{~s}$, for a total exposure time of $\simeq 17.4 \mathrm{ks}$. We used slit widths of 0.9 and 1.0 arcsec in the UVB

\footnotetext{
${ }^{1}$ Provided by The Space Telescope Science Institute (https : / github.com/spacetelescope)
} 
and visible (VIS), respectively, yielding corresponding velocity resolutions of $\sim 51,33 \mathrm{~km} \mathrm{~s}^{-1}$. The data set was reduced using the standard ESO pipeline ESOREFLEX ${ }^{45}$ version 3.3.5. Calibration frames were taken every $1 \mathrm{~h}$ and additionally during the occultation of HST by the Earth. A total of 20 science exposures of five minutes long were gathered with GTC/OSIRIS, covering the first two hours of the campaign, using the grism R2500R $(\lambda \lambda 5575-7685 \AA)$ and one with R1000B $(\lambda \lambda 4200-$ $7400 \AA$ ) , delivering a velocity resolution of $\sim 160 \mathrm{~km} \mathrm{~s}^{-1}$ and $\sim 350 \mathrm{~km} \mathrm{~s}^{-1}$ respectively. Further details on the data reduction of these observation are given in Muñoz-Darias et al. $(2020)^{2}$.

\section{Outflow diagnostics}

The presence of blue-shifted absorption associated with far-UV and optical lines implies a significant column density of material in the lower level of the relevant atomic transition. This, in turn, can be used to set a rough lower limit on the mass-loss rate of the outflow.

Following ${ }^{46}$, we approximate the outflow as spherical and adopt a simple Hubble-like $v \propto R$ velocity law. Combining the expression for the Sobolev optical depth with the continuity equation, the characteristic optical depth presented by such an outflow at velocity $v$ in a given line can be written as

$$
\tau \simeq 74.1\left(\frac{f_{\text {osc }}}{0.2847}\right)\left(\frac{\lambda}{1549.062 \AA}\right)\left(\frac{A}{7 \times 10^{-5}}\right)\left(\frac{f_{\text {ion }}}{1.00}\right)\left(\frac{\dot{M}_{w}}{10^{-10} \mathrm{M}_{\odot} \mathrm{yr}^{-1}}\right)\left(\frac{v}{1500 \mathrm{~km} \mathrm{~s}^{-1}}\right)^{-2}\left(\frac{R(v)}{10^{10} \mathrm{~cm}}\right)^{-1} .
$$

Here, $f_{\text {osc }}$ and $\lambda$ are the oscillator strength and wavelength of the line, respectively, $A$ is the abundance of the relevant element, $f_{\text {ion }}$ is the fraction of those atoms in the correct ionization level, $\dot{M}_{w}$ is the mass-loss rate of the outflow, and $R(v)$ is the radius where velocity $v$ is reached in the wind.

The reference values adopted for $f_{\text {osc }}, \lambda$ and $A$ in Equation 2 are representative of the $\mathrm{C}$ IV resonance line (treated as a singlet). The reference velocity, $v \simeq 1500 \mathrm{~km} \mathrm{~s}^{-1}$, is chosen based on the location of the blue-shifted absorption trough in the far-UV line profiles (cf Figure 4). Our adopted value of $R(v) \simeq 10^{10} \mathrm{~cm}$ corresponds to the radius in the disc beyond which a thermally driven outflow is expected to be launched (see below); it is also roughly the radius where $v_{\mathrm{esc}} \simeq 1500 \mathrm{~km} \mathrm{~s}^{-1}$. Finally, by taking $f_{\text {ion }}=1$, we ensure that our estimate of $\dot{M}_{w}$ is a lower limit (modulo uncertainties in the other parameters).

Based on the depth of the absorption features in the far-UV line profiles, we expect that $\tau \gtrsim 1$ for both $\mathrm{N} V$ and $\mathrm{C}$ IV. The estimated lower limits on the mass-loss rates are then $\dot{M}_{w} \gtrsim 2 \times 10^{-11}$ from N v and $\dot{M}_{w} \gtrsim 3 \times 10^{-12}$ from C IV.

\section{A thermally driven disc wind in Swift J1858.6-0814?}

The accretion discs in luminous X-ray binaries are subject to strong irradiation. As a result, the upper layers of the atmosphere can be heated to the inverse Compton temperature, which depends only on the spectral energy distribution of the radiation field. The X-ray spectrum of $S$ wift J1858.6-0814 in the hard state can be approximated as a power law with photon index $\Gamma=1.5$ and an exponential cut off at $E_{\max } \simeq 30 \mathrm{keV}^{28}$. For such a spectrum, the Compton temperature is approximately $k T_{I C} \simeq E_{\max } / 12^{47}$, which gives $T_{I C} \simeq 3 \times 10^{7} \mathrm{~K}$ for $S$ wift J1858.6-0814.

Mass loss from these heated layers is inevitable at radii where the characteristic thermal speed of the ions, $v_{\text {th }} \simeq 3 k T_{I C} / m_{p}$ exceeds the local escape velocity, $v_{\mathrm{esc}}=2 G M / R$. Discs larger than the so-called Compton radius, $R_{I C}=\left(2 G M m_{p}\right) /\left(3 k T_{I C}\right)$, are therefore expected to produce thermally driven outflows. For $S$ wift J1858.6-0814, we obtain $R_{I C} \simeq 5 \times 10^{10} \mathrm{~cm}$. In reality, the radius at which this mechanism turns on is typically $R_{\min } \simeq 0.1 R_{I C}{ }^{48}$. In our mass-loss rate calculation above, we have adopted a characteristic radius $R \simeq 0.3 R_{I C}$ for the line-forming region in the outflow.

The disc in Swift J1858.6-0814 is certainly large enough to drive such an outflow. The orbital period of the system is $P_{\text {orb }} \simeq 21.3 \mathrm{~h}^{13}$. From Kepler's third law, and assuming that $q=M_{2} / M_{1} \lesssim 1$, the binary separation is $a_{\text {bin }} \simeq 3 \times 10^{11} \mathrm{~cm}$. If the disc is tidally limited, its outer radius will be roughly $R_{\text {disc }} \simeq 0.9 R_{1}$, where $R_{1}$ is the Roche-lobe radius of the neutron star ${ }^{49}$. The outer disc radius is therefore expected to be $R_{\text {disc }} \simeq 1-2 \times 10^{11} \mathrm{~cm}$ - much larger than $R_{I C}$, let alone $R_{\min } \simeq 0.1 R_{I C}$.

The final condition for strong thermally driven mass loss is that the irradiating luminosity should be sufficiently strong, $L \gtrsim L_{c r i t}=0.05 L_{E d d}{ }^{24}$. This is comparable to the time-averaged luminosity in the flaring hard state of Swift J1858.6-081422. It is therefore likely that the system was luminous enough to drive a powerful thermal disc wind.

\section{References}

1. Ponti, G. et al. Ubiquitous equatorial accretion disc winds in black hole soft states. MNRAS 422, L11-L15, DOI: 10.1111/j.1745-3933.2012.01224.x (2012). 1201.4172.

2. Muñoz-Darias, T. et al. The Changing-look Optical Wind of the Flaring X-Ray Transient Swift J1858.6-0814. ApJ 893, L19, DOI: 10.3847/2041-8213/ab8381 (2020). 2003.12073.

3. Long, K. S. \& Knigge, C. Modeling the Spectral Signatures of Accretion Disk Winds: A New Monte Carlo Approach. ApJ 579, 725-740, DOI: 10.1086/342879 (2002). astro-ph/0208011. 
4. Higginbottom, N. et al. Thermal and radiation driving can produce observable disc winds in hard-state X-ray binaries. MNRAS 492, 5271-5279, DOI: 10.1093/mnras/staa209 (2020). 2001.08547.

5. Tetarenko, B. E., Lasota, J. P., Heinke, C. O., Dubus, G. \& Sivakoff, G. R. Strong disk winds traced throughout outbursts in black-hole X-ray binaries. Nature 554, 69-72, DOI: 10.1038/nature25159 (2018). 1801.07203.

6. Tetarenko, B. E., Dubus, G., Marcel, G., Done, C. \& Clavel, M. Thermally driven disc winds as a mechanism for X-ray irradiation heating in black hole X-ray binaries: the case study of GX339-4. MNRAS 495, 3666-3682, DOI: 10.1093/mnras/staa1367 (2020). 2005.06378.

7. Gehrels, N. et al. The Swift Gamma-Ray Burst Mission. ApJ 611, 1005-1020, DOI: 10.1086/422091 (2004). astro-ph/ 0405233.

8. Krimm, H. A. et al. Swift reports the detection of a new galactic transient source Swift J1858.6-0814. The Astron. Telegr. 12151, 1 (2018).

9. Bright, J. et al. AMI-LA radio observations of the galactic X-ray transient Swift J1858.6-0814. The Astron. Telegr. 12184, 1 (2018).

10. Vasilopoulos, G., Bailyn, C. \& Milburn, J. Swift J1858.6-0814: Localization and variability of the optical counterpart. The Astron. Telegr. 12164, 1 (2018).

11. Kennea, J. A. \& Krimm, H. A. Swift J1858.6-0814: Swift XRT and UVOT localization. The Astron. Telegr. 12160, 1 (2018).

12. Buisson, D. J. K. et al. Discovery of thermonuclear (Type I) X-ray bursts in the X-ray binary Swift J1858.6-0814 observed with NICER and NuSTAR. MNRAS 499, 793-803, DOI: 10.1093/mnras/staa2749 (2020). 2009.03334.

13. Buisson, D. J. K. et al. Dips and eclipses in the X-ray binary Swift J1858.6-0814 observed with NICER. MNRAS 503, 5600-5610, DOI: 10.1093/mnras/stab863 (2021). 2103.12787.

14. Baglio, M. C. et al. Optical observations of the rapidly varying newly discovered transient Swift J1858.6-0814. The Astron. Telegr. 12180, 1 (2018).

15. Paice, J. A. et al. Blue Oscillations and Rapid Red Flares in Swift J1858.6-0814 Observed with ULTRACAM/NTT. The Astron. Telegr. 12197, 1 (2018).

16. Rajwade, K. et al. More optical follow-up of Swift J1858.6-0814. The Astron. Telegr. 12186, 1 (2018).

17. Ludlam, R. M. et al. Initial NICER observation of the new X-ray transient Swift J1858.6-0814. The Astron. Telegr. 12158, 1 (2018).

18. Reynolds, M. T., Miller, J. M., Ludlam, R. M. \& Tetarenko, B. E. Significant Intrinsic Absorption in Swift J1858.6-0814. The Astron. Telegr. 12220, 1 (2018).

19. Kimura, M. et al. Repetitive patterns in rapid optical variations in the nearby black-hole binary V404 Cygni. Nature 529, 54-58, DOI: 10.1038/nature16452 (2016). 1607.06195.

20. Motta, S. E., Kajava, J. J. E., Sánchez-Fernández, C., Giustini, M. \& Kuulkers, E. The black hole binary V404 Cygni: a highly accreting obscured AGN analogue. MNRAS 468, 981-993, DOI: 10.1093/mnras/stx466 (2017). 1607.02255.

21. Motta, S. E. et al. Swift observations of V404 Cyg during the 2015 outburst: X-ray outflows from super-Eddington accretion. MNRAS 471, 1797-1818, DOI: 10.1093/mnras/stx1699 (2017). 1707.01076.

22. Hare, J. et al. NuSTAR Observations of the Transient Galactic Black Hole Binary Candidate Swift J1858.60814: A New Sibling of V404 Cyg and V4641 Sgr? ApJ 890, 57, DOI: 10.3847/1538-4357/ab6a12 (2020). 2001.03214.

23. van den Eijnden, J. et al. The variable radio counterpart of Swift J1858.6-0814. MNRAS 496, 4127-4140, DOI: 10.1093/mnras/staa1704 (2020). 2006.06425.

24. Begelman, M. C., McKee, C. F. \& Shields, G. A. Compton heated winds and coronae above accretion disks. I. Dynamics. ApJ 271, 70-88, DOI: 10.1086/161178 (1983).

25. Done, C., Gierliński, M. \& Kubota, A. Modelling the behaviour of accretion flows in X-ray binaries. Everything you always wanted to know about accretion but were afraid to ask. A@AND@ARev 15, 1-66, DOI: 10.1007/s00159-007-0006-1 (2007). 0708.0148.

26. Higginbottom, N. \& Proga, D. Coronae and Winds from Irradiated Disks in X-Ray Binaries. ApJ 807, 107, DOI: 10.1088/0004-637X/807/1/107 (2015). 1504.03328. 
27. Díaz Trigo, M. \& Boirin, L. Accretion disc atmospheres and winds in low-mass X-ray binaries. Astron. Nachrichten 337, 368, DOI: 10.1002/asna.201612315 (2016). 1510.03576.

28. Buisson, D. J. K. et al. Soft X-ray emission lines in the X-ray binary Swift J1858.6-0814 observed with XMM-Newton Reflection Grating Spectrometer: disc atmosphere or wind? MNRAS 498, 68-76, DOI: 10.1093/mnras/staa2258 (2020). 2007.14407.

29. Prinja, R. K., Ringwald, F. A., Wade, R. A. \& Knigge, C. HST ultraviolet observations of rapid variability in the accretion-disc wind of BZ Cam. MNRAS 312, 316-326, DOI: 10.1046/j.1365-8711.2000.03111.x (2000).

30. Muñoz-Darias, T. et al. Regulation of black-hole accretion by a disk wind during a violent outburst of V404 Cygni. Nature 534, 75-78, DOI: 10.1038/nature17446 (2016). 1605.02358.

31. Shields, G. A., McKee, C. F., Lin, D. N. C. \& Begelman, M. C. Compton-heated Winds and Coronae above Accretion Disks. III. Instability and Oscillations. ApJ 306, 90, DOI: 10.1086/164322 (1986).

32. Ioannou, Z. et al. Understanding the LMXB X2127+119 in M 15. II. The UV data. A@AND@A 399, 211-218, DOI: 10.1051/0004-6361:20021578 (2003). astro-ph/0212127.

33. Bayless, A. J., Robinson, E. L., Hynes, R. I., Ashcraft, T. A. \& Cornell, M. E. The Structure of the Accretion Disk in the Accretion Disk Corona X-ray Binary 4U 1822-371 at Optical and Ultraviolet Wavelengths. ApJ 709, 251-262, DOI: 10.1088/0004-637X/709/1/251 (2010). 0911.4492.

34. Allen, J. L. et al. The Disk Wind in the Neutron Star Low-mass X-Ray Binary GX 13+1. ApJ 861, 26, DOI: 10.3847/ 1538-4357/aac2d1 (2018). 1806.08800.

35. Neilsen, J. et al. A NICER View of a Highly Absorbed Flare in GRS 1915+105. ApJ 902, 152, DOI: 10.3847/1538-4357/ abb598 (2020). 2010.14512.

36. Lasota, J.-P. Black Hole Accretion Discs, vol. 440 of Astrophysics and Space Science Library, 1 (2016).

37. Dubus, G., Hameury, J. M. \& Lasota, J. P. The disc instability model for X-ray transients: Evidence for truncation and irradiation. A@AND@A 373, 251-271, DOI: 10.1051/0004-6361:20010632 (2001). astro-ph/0102237.

38. Coriat, M., Fender, R. P. \& Dubus, G. Revisiting a fundamental test of the disc instability model for X-ray binaries. MNRAS 424, 1991-2001, DOI: 10.1111/j.1365-2966.2012.21339.x (2012). 1205.5038.

39. Charles, P. et al. Hot, dense He II outflows during the 2017 outburst of the X-ray transient Swift J1357.2-0933. MNRAS 489, L47-L52, DOI: 10.1093/mnrasl/slz120 (2019). 1908.00320.

40. Higginbottom, N., Proga, D., Knigge, C. \& Long, K. S. Thermal Disk Winds in X-Ray Binaries: Realistic Heating and Cooling Rates Give Rise to Slow, but Massive, Outflows. ApJ 836, 42, DOI: 10.3847/1538-4357/836/1/42 (2017). 1612.08996.

41. Green, J. C. et al. The Cosmic Origins Spectrograph. ApJ 744, 60, DOI: 10.1088/0004-637X/744/1/60 (2012). 1110.0462.

42. Eracleous, M. \& Horne, K. The Speedy Magnetic Propeller in the Cataclysmic Variable AE Aquarii. ApJ 471, 427, DOI: 10.1086/177979 (1996).

43. Vernet, J. et al. X-shooter, the new wide band intermediate resolution spectrograph at the ESO Very Large Telescope. $A @ A N D @ A$ 536, A105, DOI: 10.1051/0004-6361/201117752 (2011). 1110.1944.

44. Cepa, J. et al. OSIRIS tunable imager and spectrograph. In Iye, M. \& Moorwood, A. F. (eds.) Optical and IR Telescope Instrumentation and Detectors, vol. 4008 of Society of Photo-Optical Instrumentation Engineers (SPIE) Conference Series, 623-631, DOI: 10.1117/12.395520 (2000).

45. Freudling, W. et al. Automated data reduction workflows for astronomy. The ESO Reflex environment. A@AND@A 559, A96, DOI: 10.1051/0004-6361/201322494 (2013). 1311.5411.

46. Drew, J. E. Inclination and orbital-phase-dependent resonance line-profile calculations applied to cataclysmic variable winds. MNRAS 224, 595-632, DOI: 10.1093/mnras/224.3.595 (1987).

47. Done, C. Observational characteristics of accretion onto black holes. arXiv e-prints arXiv:1008.2287 (2010). 1008.2287.

48. Done, C., Tomaru, R. \& Takahashi, T. Thermal winds in stellar mass black hole and neutron star binary systems. MNRAS 473, 838-848, DOI: 10.1093/mnras/stx2400 (2018). 1612.09377.

49. Frank, J., King, A. \& Raine, D. J. Accretion Power in Astrophysics: Third Edition (2002).

50. Ashman, K. M., Bird, C. M. \& Zepf, S. E. Detecting Bimodality in Astrometrical Datasets. AJ 108, 2348, DOI: 10.1086/117248 (1994). astro-ph/9408030. 

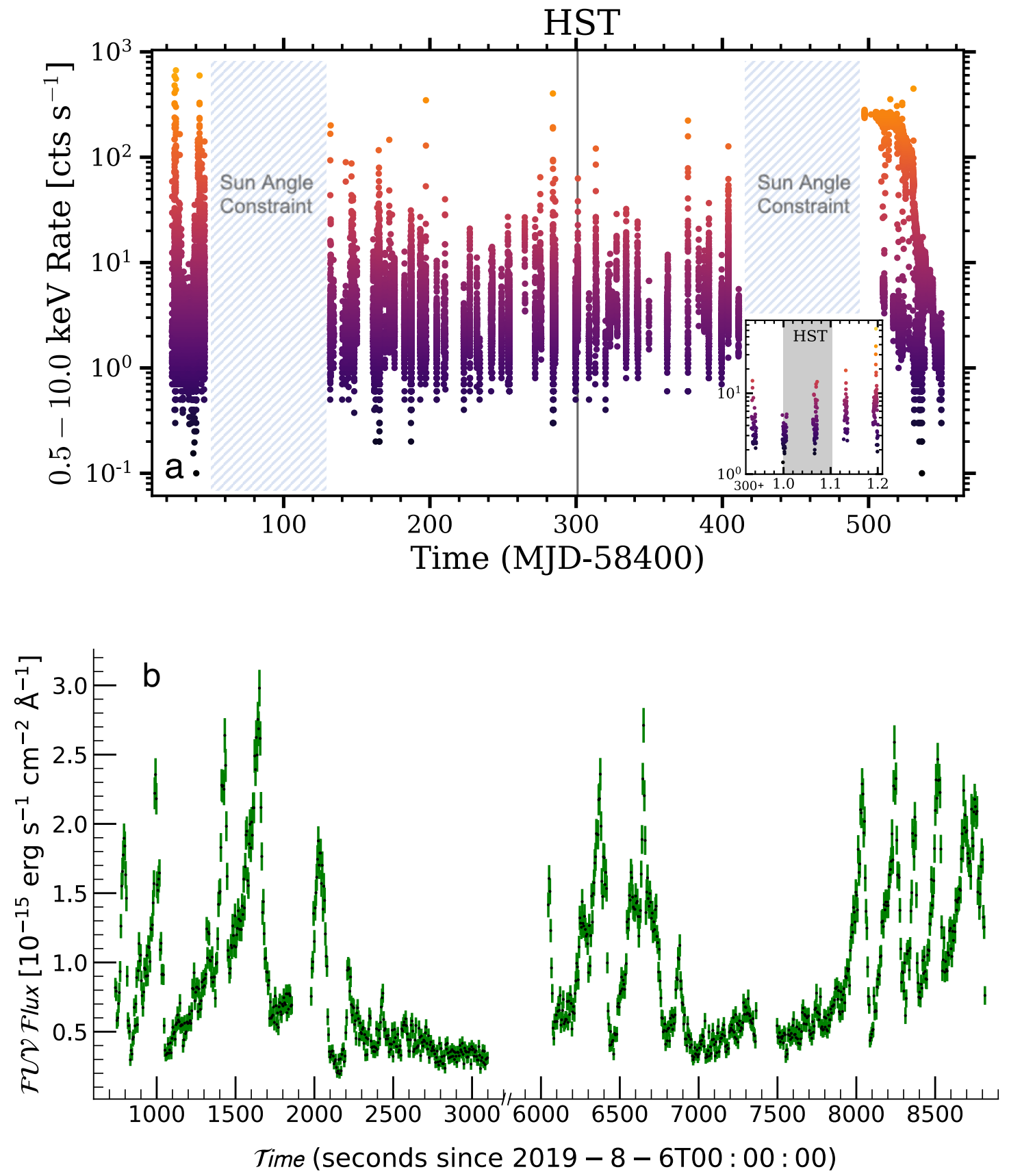

Figure 1. Top: Overview light curves of the $X$-ray transient $S$ wift J1858.6-0814 . (a) Outburst evolution as observed with NICER in the $0.5-10 \mathrm{keV}$ band (reproduced from Buisson et al. $(2020)^{13}$ ); the two large gaps are caused by Sun constraints. The source exhibits flares that reach the Eddington limit during the first 450 days while it is in the hard state. The time of the HST far-UV observations is marked with a vertical line. Colour code refers to the observed count rate. Inset shows a zoom-in around the time of the HST observations (MJD $\simeq 58701$ ), indicated with the shaded area. The inset cover the region around the time of the HST visit. (b) HST far-UV light-curve in 5s bins, showing strong flares (up to a factor of 10 increase in flux) and flickering at lower flux levels. 


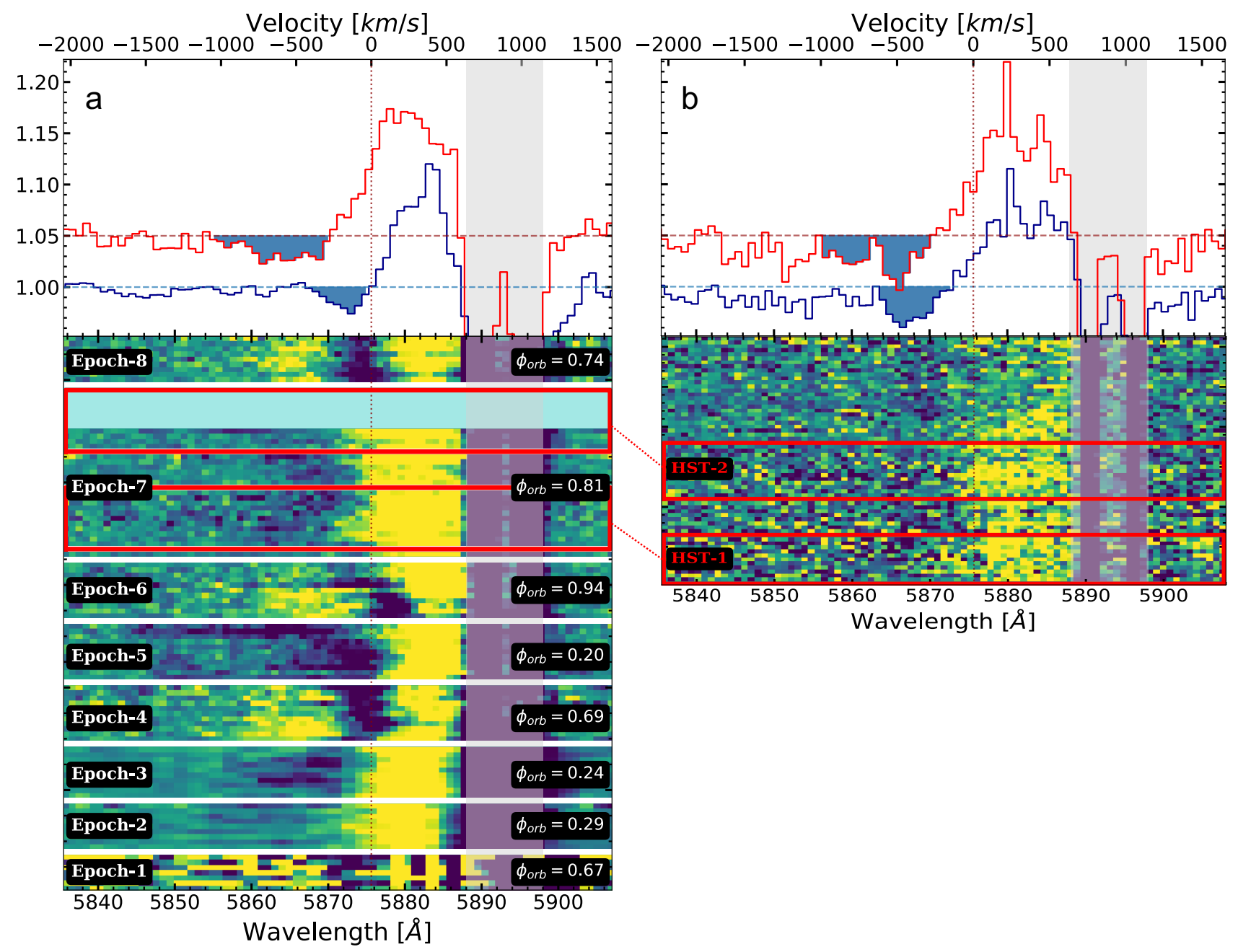

Figure 2. Apparently transient optical wind signatures. (a) Trailed spectra of the 8 GTC/OSIRIS epochs published in Muñoz-Darias et al. (2020) ${ }^{2}$ with the corresponding orbital phase ${ }^{13}$ and (b) VLT/X-Shooter during the HST visit (right) centered on He I $5876 \AA$. The average spectrum of all the observations is shown in the top panel with a blue line. Strictly simultaneous observations during the two ultraviolet exposures are highlighted in red boxes, with their corresponding averaged spectrum shown in red in the top panels with a 5\% offset for clarity. Absorption troughs below continuum levels are highlighted with a shaded area. Telluric absorption region around $\lambda 5836 \AA ̊$ is indicated with the shaded vertical band. 


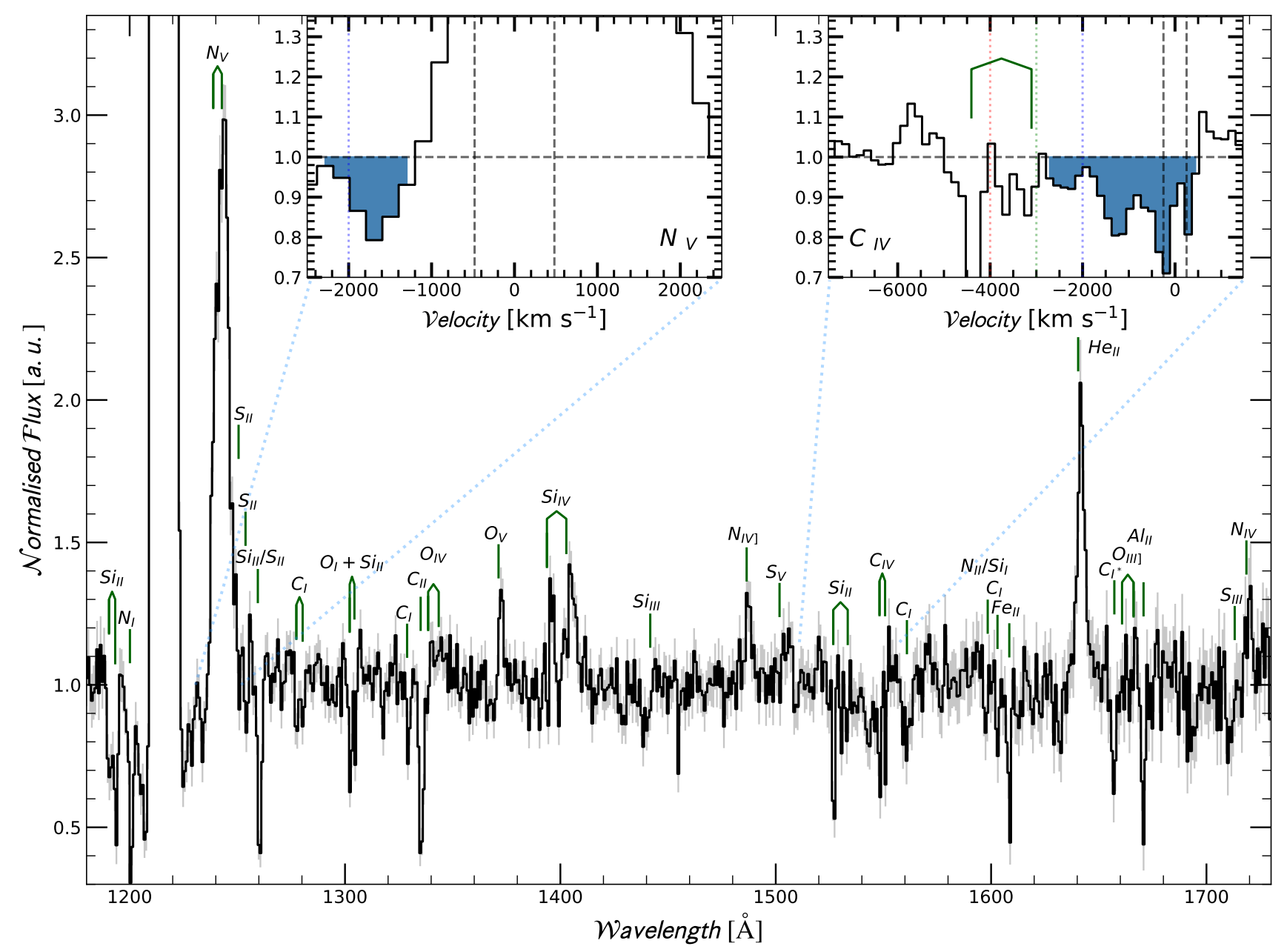

Figure 3. Average far-UV spectrum of $S$ wift J1858.6-0814 during the luminous hard state. Numerous emission and absorption lines are present; the dominant transitions have been labeled with their corresponding rest position indicated with a green tick. All the emission components are skewed toward shorter wavelengths with blue absorption troughs, which are the characteristic footprint of disc outflows. Insets show a zoom-in to the $N v(\lambda \lambda 1284-1437 \AA)$ and $C$ IV $(\lambda \lambda 1513-1668 \AA)$ profiles with the blue-shifted absorption signatures highlighted in blue, in the latter nearby Si II interstellar absorption is indicated with connected green ticks. These signatures indicate the presence of a warm, moderately ionized accretion disc wind with characteristic velocities similar to those observed in the optical. 


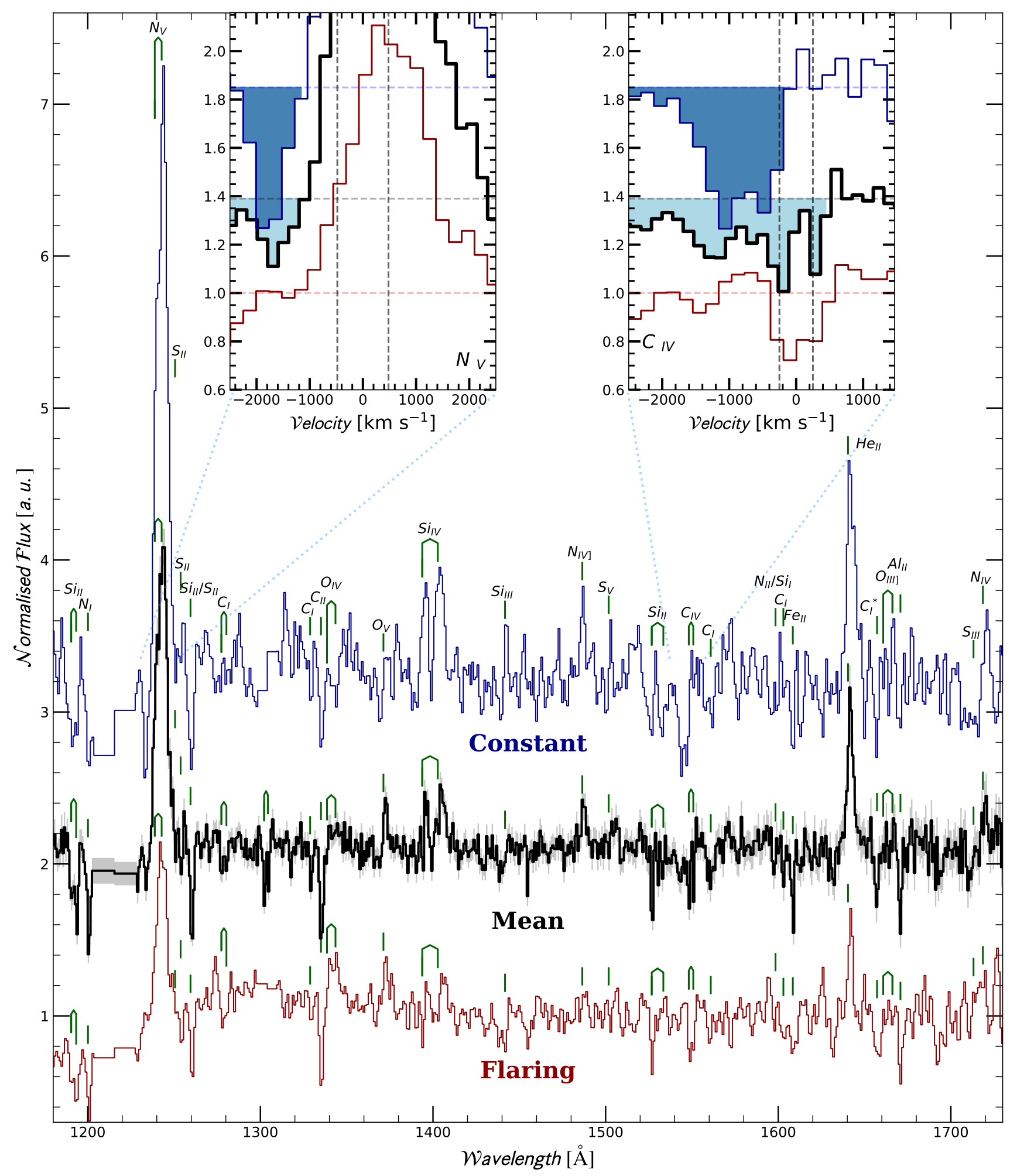

Figure 4. Spectral decomposition into a constant (blue) and flaring component (red). Being the latter driven by the observed continuum variability in the far-UV. The average normalized spectrum is displayed with a thick black line for reference, and all are normalized to the continuum level. An offset has been added to the spectra for clarity. The regions of geocoronal emission like Lyman $\alpha$ and Si II were removed to avoid artifacts in the spectral decomposition. Rest positions of the dominant ions are marked with a green tick and labeled in the top spectrum. Insets are zooms into the two transitions in which the presence of the outflow is more prominent. Specifically, regions covered in the insets are $\lambda \lambda 1284-1474 \AA$ for N V and $\lambda \lambda 1525-1717 \AA$ for $\mathrm{C}$ IV. 


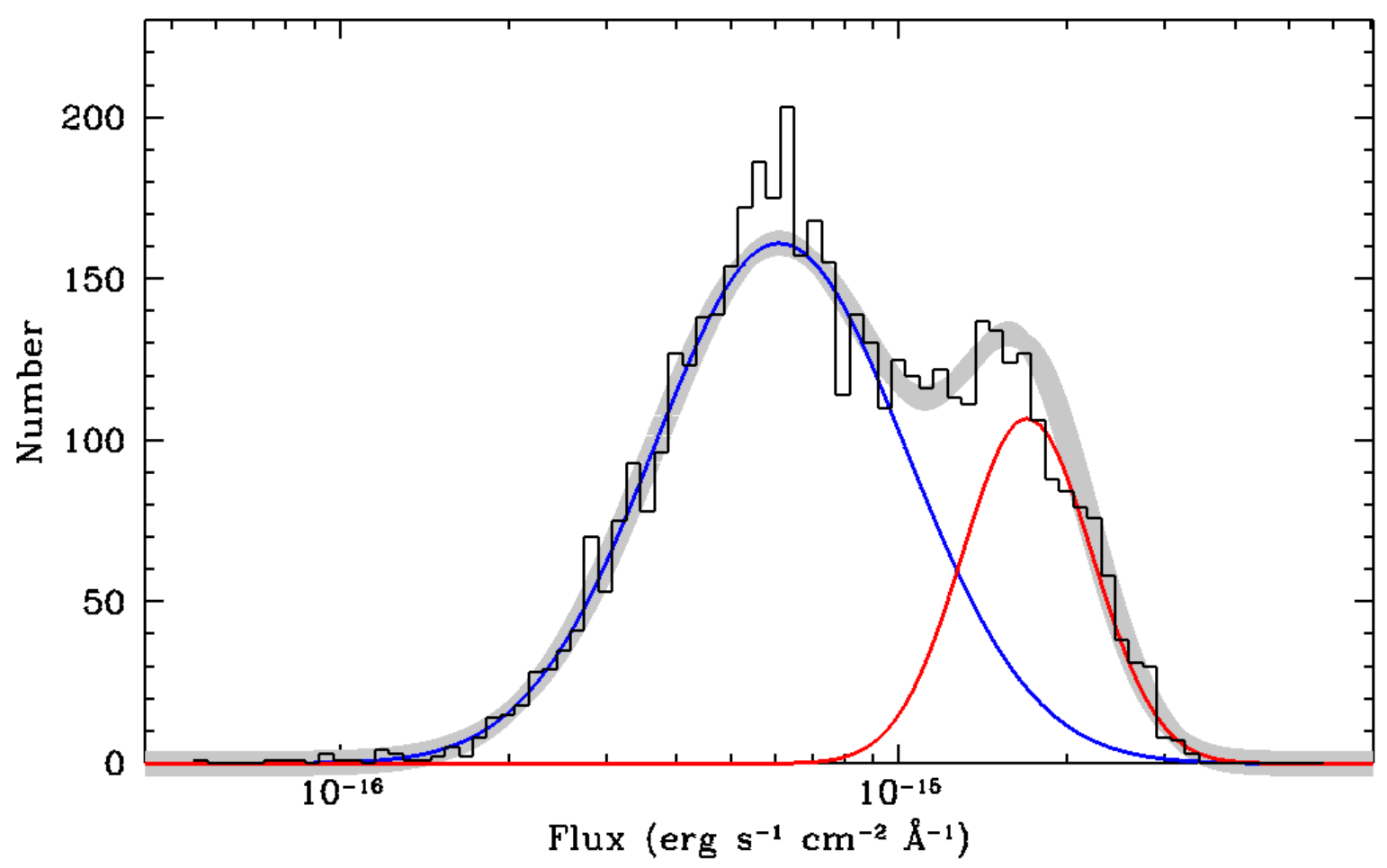

Extended Data Figure 1. The logarithmic far-UV flux distribution of J1858 during our observations. The distribution is clearly bimodal, consistent with the visual impression from the light curve (Figure 1 lower panel) of the variability being due to a flaring component that is superposed on a roughly constant component. The grey line is the optimal decomposition of the distribution into two Gaussians, as suggested by the KMM algorithm ${ }^{50}$. The blue and red lines correspond to the individual Gaussians. KMM rejects the null hypothesis of a single component with extremely high significance $\left(p<10^{-43}\right)$.

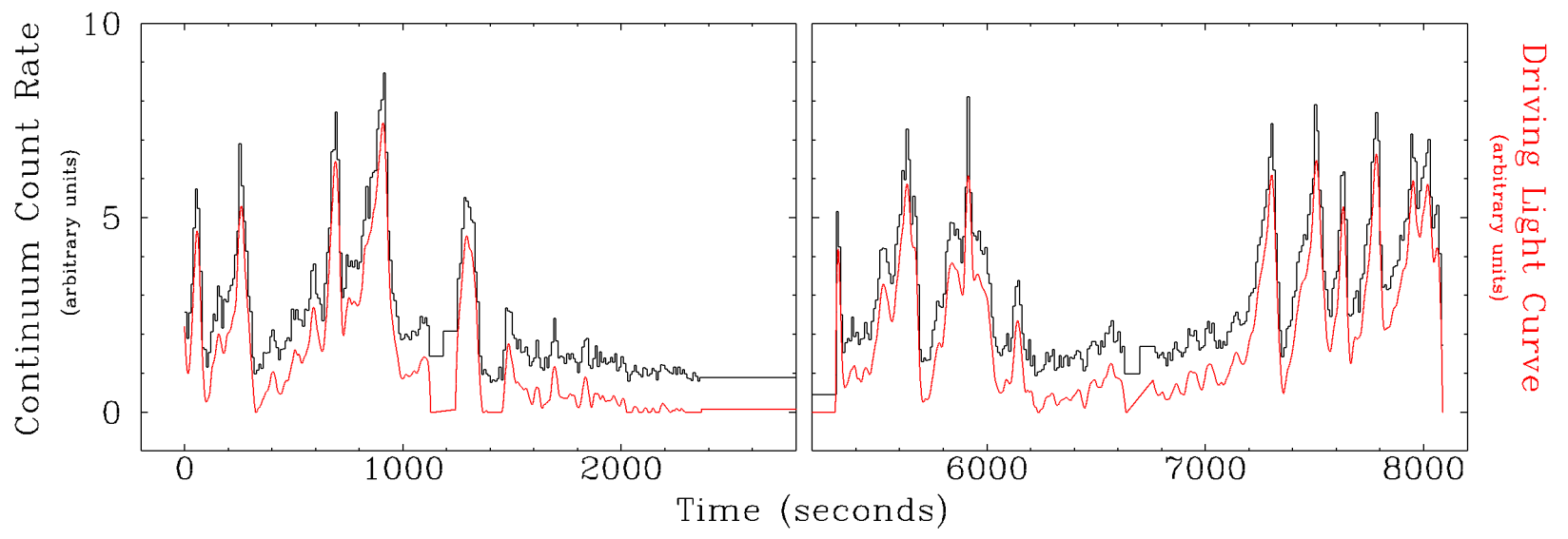

Extended Data Figure 2. The far-UV continuum and driving light curves. The black histogram shows the light curve of $S$ wift J1858.6-0814 constructed from three broad wavelength regions that exclude the three strongest emission lines (N v $\lambda 1240$, Si IV $\lambda 1400$ and He II $\lambda 1640)$. The specific regions used were $\lambda \lambda 1290 \AA-1390 \AA, 1410 \AA-1630 \AA, 1660 \AA-$ $1850 \AA$. The light curve is shown normalized to an estimate of the underlying constant level $\left(80 \mathrm{c} \mathrm{s}^{-1}\right)$. The driving light curve used in the decomposition, $D(t)$, was constructed from this and is shown as the red curve. It was obtained by subtracting the estimate of the constant level, setting any slightly negative values to zero, and using a 5-point, 2nd order Savitzky-Golay filter to produce a slightly smoother, higher $\mathrm{S} / \mathrm{N}$ version of the light curve. 